\title{
Omni-Directional Display System for Group Activity on Life Review Therapy
}

\author{
Tomoyuki Honda and Kunio Sakamoto \\ Department of Intelligence and Informatics, Konan University \\ 8-9-1 Okamoto, Higashinada, Kobe 658-8501, Japan \\ kunio@konan-u.ac.jp
}

\begin{abstract}
The authors have researched support system of the reminiscence and life review activity. This support system consists of an interactive tabletop display and interface system. On the reminiscence and life review activity, a therapist puts pictures on the table so as to trigger a talk. However some observers may perceive upside down images if they sit down opposite the therapist. To overcome this problem, we have developed the display system which can be viewed from any direction. In this paper, we propose a 4-views tabletop flat display system for cooperative activity on a round table.
\end{abstract}

Keywords: all around viewing, group work, viewing angle control film, tabletop display.

\section{Introduction}

The authors have researched multimedia system and support system for nursing studies on and practices of reminiscence therapy and life review therapy. The concept of the life review is presented by Butler in 1963. The process of thinking back on one's life and communicating about one's life to another person is called life review. A therapist must keep a record of sessions for inspection of methods and ways of valuation on reminiscence and life review therapy, but it is trouble for the therapist to record. The aim of research is to develop the support system which can automatically give an optimum topic and write down a session report about the activity. This life review is often assisted by aids such as videos, pictures, objects, archives and life story books, as shown in fig. 1, in order to make an opportunity of talking. We want to develop an omni-directional display system for cooperative activity on a round table to enable all-around viewing and unification of media contents by an electronic form.

\section{Motivations}

This paper describes an omni-directional display system that can be viewed from any direction (i.e., the display has four viewing zones so as to perceive a screen view of the display at all directions around a table). The authors have ever researched information display systems involving 3D imaging. However, a conventional monitor 
display is viewed from one direction, that is, the display has narrow viewing angle and observers cannot view the screen from the opposite side. Hence we developed a 4 -views display system for collaborative tasks cooperated by four users. This 4 -views display can provide different images to four users surrounding the system utilizing an optical grating film for generating a virtual screen and floating above the top level of an actual display panel. The floating virtual screens of this display are generated into a square pyramid in front of observers' eyes. But this system displays different images in the pyramid and the display screen is not flat. Then this paper describes a flat type display system for the all-around viewing, which enables to put something on the table.

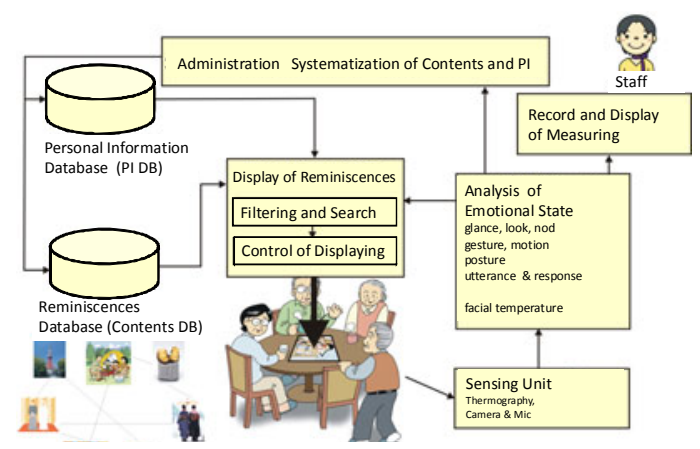

Fig. 1. Cooperative work of life review activity

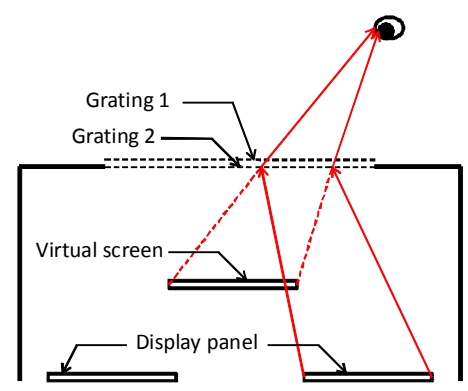

Fig. 2. Optical layout of display

\section{4-Views Tabletop Flat Display}

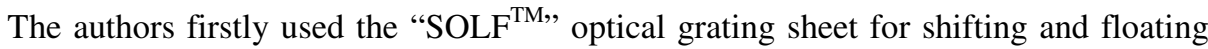
image planes above the top level of an actual display panel. This display system is the shape where the outer surfaces are triangular and converge at a point. We would like to shape a new display like a conventional table. To enable all-around viewing from four directions, it is necessary to generate a virtual screen. So this viewscreen is floating above an actual display panel and sinking below the level of the table as shown in fig. 2. To simplify an optical layout, the authors utilize a special grating sheet for locating pseudo images at different places from original positions. The author unexpectedly found this special sheet at the Sannomiya store of Tokyu Hands. As shown in fig. 3 , this grating sheet provides a diffracted image which is arranged left or right from original position. The grating sheet diffracts or scatters a light beam with a designed angle. This interesting phenomenon reminds us of method to shift image positions by a simple optical layout. This product name is unknown. We regard this sheet as a cheeper copy product of the SOLF sheet. However this sheet has a useful characteristic. Using this film, you can observe shifted image of the left card from the left, but not from the right as shown in fig 3. The right card is also observable as the same. Thus this new grating film has an effect of the viewing angle control in addition unlike an original SOLF sheet.

As shown in fig. 2, our developed omni directional display system consists of four LCD panels and two optical grating films for shifting original images into the center 
and separating virtual images into an appropriate eye. As above mentioned, one grating sheet can shift left and right images into the center and deliver each image to an observer. To generate four virtual screens, we piled up two grating sheets. The important point is that each sheet is inclined at 45 degrees to an edge of the table because both sheets shift the images into any direction independently. As shown in fig. 4, the combination of two optical sheets generates four directional image shifts. Each grating sheet shifts an actual image into any direction at 45 degrees to horizontal. Both image shifts makes vertical shift as the result. Other image shifts are also generated just the same. Fig 5 shows the appearance of our developed prototype display. Four display panels are arrayed crosswise in order to produce four virtual image screens into the center. A grating film appropriately locates apart from the panels so as to shift images into the center for delivering an appropriate image into each observer by adjusting the interval between the optical sheet and the display panels. The lights of an LCD pass through the films or are blocked by the films whether eyes are corresponding to each image or not. An observer can perceive delivered image without wearing special glasses.

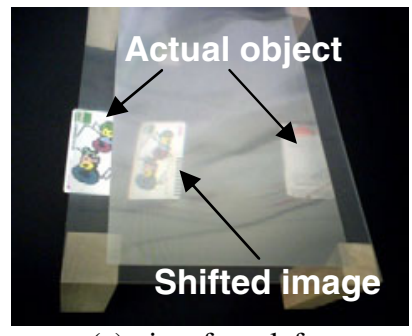

(a) view from left

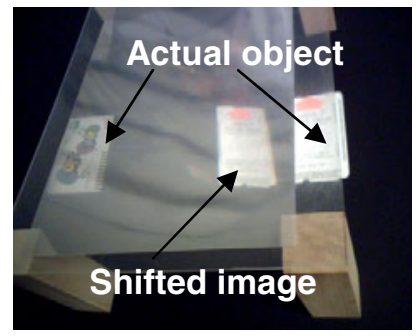

(b) view from right

Fig. 3. Image shift by grating sheet

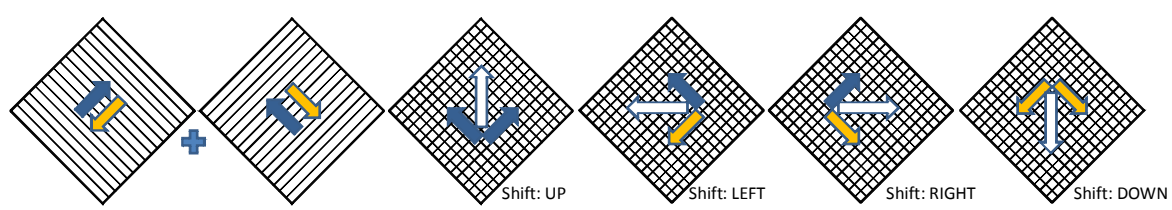

Fig. 4. Effect of image shift to four directions

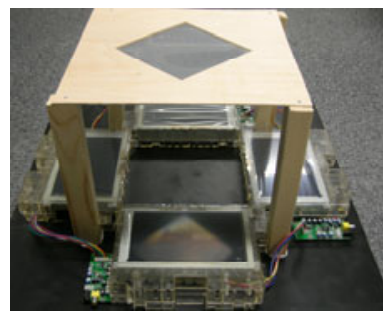

Fig. 5. Appearance of display (KNB-20) 
We have developed the prototype display system using four commercial LCD panels. These displays have a 7.24 inch LCD panel and its size is $160 \mathrm{~mm}(\mathrm{~W}) \mathrm{x}$ $90 \mathrm{~mm}(\mathrm{H})$. The video input supports NTSC. The panels are fixed on edges of a $220 \mathrm{~mm}$ square. The size of a viewing window on surfaces of the table is $150 \mathrm{~mm}$ square. This viewing window is with two grating sheets. The distance between these LCD panels and the viewing window is approximately $220 \mathrm{~mm}$ so as to shift all images into the center. Since the grating sheet not only shifts an image but also controls a viewing angle, an observer perceives an only virtual screen right in front of his/her face. Observers can watch the screen of a display from any direction because this system has four screens with four viewing directions.

Acknowledgments. This research is partially supported by "Grant-in-Aid for Young Scientists(B)" \#20700112 and "Scientific Research (C) (General)" \#20500481 from Ministry of Education, Culture, Sports, Science and Technology Japan(MEXT). 